\title{
Construction and Simulation of Athlete's Wrong Action Recognition Model in Sports Training Based on Embedded Wireless Communication and Computer Vision
}

\author{
Naichun Gao \\ Department of Sports Science, College of Education, Zhejiang University, Hangzhou, 310058 Zhejiang, China \\ Correspondence should be addressed to Naichun Gao; beckync.gao@zju.edu.cn
}

Received 14 October 2021; Revised 24 November 2021; Accepted 3 December 2021; Published 23 December 2021

Academic Editor: Haibin Lv

Copyright (c) 2021 Naichun Gao. This is an open access article distributed under the Creative Commons Attribution License, which permits unrestricted use, distribution, and reproduction in any medium, provided the original work is properly cited.

\begin{abstract}
Embedded networking has a broad prospect. Because of the Internet and the rapid development of PC skills, computer vision technology has a wide range of applications in many fields, especially the importance of identifying wrong movements in sports training. To study the computer vision technology to identify the wrong movement of athletes in sports training, in this paper, a hidden Markov model based on computer vision technology is constructed to collect video and identify the landing and take-off movements and badminton serving movements of a team of athletes under the condition of sports training, Bayesian classification algorithm to analyze the acquired sports training action data, obtain the error frequency, and the number of errors of the landing jump action, and the three characteristic data of the displacement, velocity, and acceleration of the body's center of gravity of the athlete in the two cases of successful and incorrect badminton serve actions and compared and analyzed the accuracy of the action recognition method used in this article, the action recognition method based on deep learning and the action recognition method based on EMG signal under 30 experiments. The training process of deep learning is specifically split into two stages: 1st, a monolayer neuron is built layer by layer so that the network is trained one layer at a time; when all layers are fully trained, a tuning is performed using a wake-sleep operation. The final result shows that the frequency of the wrong actions of the athletes on the landing jump is concentrated in the knee valgus, the total frequency of error has reached 58\%, and the frequency of personal error has reached $45 \%$; the problem of the landing distance of the two feet of the team athletes also appeared more frequently, the total frequency reached $50 \%$, and the personal frequency reached $30 \%$. Therefore, athletes should pay more attention to the problems of knee valgus and the distance between feet when performing landing jumps; the difference in the displacement, speed, and acceleration of the body's center of gravity during the badminton serve will affect the error of the action. And the action recognition method used in this study has certain advantages compared with the other two action recognition methods, and the accuracy of action recognition is higher.
\end{abstract}

\section{Introduction}

1.1. Background Meaning. In this era of rapid development of science and technology, computer technology has gradually spread throughout people's daily studies, work, and lives. The development of computer technology is gradually changing people's thinking and living habits. As a very key technology in computer technology, computer vision has a wide range of roles in artificial intelligence, image processing, motion recognition, and other fields $[1,2]$. In this context, the field of sports has also begun to introduce computer vision technology to analyze and study the wrong action recognition of athletes in sports training. And with the continuous improvement of computer technology, there are increasing methods and models for action recognition. Bayesian algorithms and hidden Markov models have always been algorithms and models frequently used in action recognition-related research.

The basis of wireless LAN is traditional wired LAN, which is an extension and replacement of wired LAN. It realizes wireless communication through wireless hubs, wireless access nodes, wireless bridges, wireless network card, and other equipment on the basis of wired LAN. To provide better convenience to people's lives, video surveillance systems 
are now using embedded microprocessors as the platform for system building, and data transmission is carried out through the network. The scope is extremely wide, from the original 4-bit processor, the 8-bit single-chip microcomputer that is still in large-scale applications, to the latest 32-bit and 64-bit embedded CPUs that are widely favored. The 32 bits are used in this article. Among them, the characteristics of embedded microprocessors are as follows: with strong support for real time and multitasking, multitasking can be completed, and the interrupt response time is short, thereby reducing the execution time of internal code and real-time operating system to a minimum. On the basis of the existing Internet, video capture equipment is connected to it. Computer vision technology can effectively identify athletes' sports training actions by extracting computer vision features, making it more convenient for researchers to collect experimental data. And the use of hidden Markov model and Bayesian algorithm makes it more convenient for researchers to deal with experimental data, reduces the complexity of experimental calculations, and improves the recognition performance of the model. It can be said that the combination of computer vision technology and sports training error action recognition research is a breakthrough in the sports field. It can effectively improve the effectiveness and accuracy of athletes' training and can effectively judge the athletes' movements, to improve the level and performance of athletes.

1.2. Related Work. At present, a number of authors have conducted studies related to the recognition of incorrect movements in sports training. Liu et al., to simplify the feature extraction process of Human Activity Recognition (HAR) and improve the generalization ability of extracted features, proposed an algorithm based on multiscale deep convolutional neural network [3], but the experimental cost of this method is relatively high. Liu et al. proposed a new action recognition method that uses $3 \mathrm{D}$ skeletal motion data captured by the Kinect depth sensor [4], but the data collection process of this method is more difficult. Zhou et al. proposed an effective method that can extract intermediate features from the Kinect skeleton for 3D human action recognition [5], but the operation steps of this method are too complicated. Jaouedi et al. introduced a human action recognition method based on the fusion and combination of sequential visual features and moving paths [6], but the experimental data of this method is incorrect and the results are unreliable. Li et al. proposed an effective framework that can recognize actions for the $3 \mathrm{D}$ skeletal kinematics joint model in less calculation time [7], but the experimental data of this method is too large. Yu et al. proposed a novel action recognition method based on deep convolutional neural network- (SP-CNN-) hierarchical pooling [8], but the data calculation process of this method is too complicated. Cui et al. proposed a motion recognition method based on depth features and motion sequences and added depth convolution information on the basis of motion units [9], but the experimental subjects of this method are too onesided. All above studies have certain shortcomings. Based on this, we have made certain improvements to the above studies, constructed a computer vision-based recognition model for athletes' sports training errors, and conducted simulation experiments.

1.3. Innovation of This Article. This article collects data on athletes' landing and jumping actions and badminton serve actions, counts the frequency of errors in landing jump actions and the displacement, speed, and acceleration of the body's center of gravity in the case of success and error in the badminton serve action, and compares and analyzes the accuracy of three different action recognition methods. The innovations of this paper are as follows: (1) using computer vision technology to collect athletes' sports training actions, (2) using hidden Markov model to identify and analyze the collected athletes' sports training actions, (3) using Bayesian algorithm to calculate and analyze the analyzed movements, and (4) using the embedded operating system; the main research is to optimize the embedded operating system, improve the operating system for specific application scenarios, and increase the support for commonly used device drivers.

\section{Related Techniques of Wrong Movement Recognition in Sports Training}

2.1. Computer Vision and Embedded Wireless Communication. Computer vision is a fast and economical nondestructive testing technology [10]; it is an important branch of motion recognition and image processing technology. Computer vision mainly simulates the visual characteristics of humans or other animals through computers and some other intelligent devices [11]. For example, industrial machine vision systems, for example, inspection bottles on the production line to accelerate through, researched as artificial intelligence and computers or robots, can understand the world around them. It can use the acquired video image as a medium to process the video image to obtain the three-dimensional information in the corresponding environment. It takes the expression and understanding of the input image as the goal to study the characteristic information of the image and then to give a computerized expression of the image content. We can use computer vision algorithms to design automated aids for human inspection tasks [12]. Computer vision uses different video image acquisition systems to replace the human visual organs, using it as an input means of visual perception, and then analyzes and processes the obtained data through the computer instead of the human brain. The ultimate research goal of computer vision is to allow computers to recognize the surrounding environment or objects in the form of human observation and understanding of the world and have the ability to adapt to the environment independently, which can only be achieved through long-term efforts. Therefore, before realizing the final research goal of computer vision, we must first establish a vision system that can complete certain tasks intelligently based on a certain degree of visual sensitivity and feedback [13]. Energy function refers to the matter to be clustered as a system, and the degree of difference between things is regarded as the energy between the elements of the system. When the energy reaches a certain 
level, the things form a new class, which means that the system needs to be reclassified.

The development of computer vision has been going on since the last century; at the beginning, MIT in the United States obtained three-dimensional structures of polyhedrons from digital images; through the analysis of two-dimensional image, the relationship between object shape and space is established, and the $3 \mathrm{D}$ scene information of the object is obtained. 2D refers to the four directions of left, right, top, and bottom, and there is no front or back side. The stuff on a paper can be considered as $2 \mathrm{D}$. It means that there is only area and absence of volume. After that, some scholars proposed a computer vision theory different from twodimensional image analysis. More specifically, it refers to the use of cameras and computers instead of human eyes to recognize, track, and measure objects. The processing becomes an image that is more suitable for human eyes to observe or transmit to the instrument for inspection. Active vision refers to the fact that the vision system allows the motion of the camera to be defined based on the available analysis and the current visual requirements, and the respective picture is obtained from the right angle. Computer vision has been widely used in all walks of life because of its advantages such as no contact with the object to be measured, wide range of observations, long-term observation, and investigation of experimental objects, and has achieved many excellent research results [14]. However, with the current development of computer vision, computer vision is still in the stage of organizing and recognizing image information, the analysis and processing of the identified object are still in the primary stage of research, and there is not much research related to it. Embedded wireless network video surveillance system is driven by the development of these technologies, and it produces an embedded system that integrates image acquisition, video compression, network transmission, and other tasks. The embedded network video surveillance system uses a high-performance MCU combined with a multitasking operating system to form an embedded application platform. After the hardware device is connected to the system through a driver in the Linux operating system, the hardware device will be in the form of a file Exist, and the system performs various operations on hardware devices in the form of accessing files. The aim of image preprocessing in this system is mainly to remove extraneous messages from images, recover useful and true messages in images, improve the measurability of pertinent messages, simplify data to the maximum extent, and thus improve the reliability of feature extraction, image segmentation, matching, and recognition. Image banalization is the process of setting the gray value of the pixels in the image to 0 or 255 , that is, the entire image presents an obvious black and white effect.

2.2. Bayesian Classification Algorithm. Bayesian classification algorithm is a classification method of statistics. It is a class of algorithms that use knowledge of probability and statistics for classification. The algorithm can be applied to large databases, and the method is simple, the classification accuracy is high, and the speed is fast.
Bayesian classification algorithm is an algorithm that uses probability and statistical knowledge to classify statistical data, and Bayesian model is a model used to process the prior probability; the final prediction result is the category with the highest probability. In the process of probability calculation, the Bayesian formula is an essential step. Suppose the sample space is $\Omega$, the randomized trial is $E$, and $A_{1}, A_{2}, \cdots, A_{n}$ is a set of event groups of $E$; for any event $B$, when $P(B)>0$, the Bayesian formula is as follows:

$$
P\left(A_{k} \mid B\right)=\frac{P\left(A_{k}\right) P\left(B \mid A_{k}\right)}{\sum_{i=1}^{n} P\left(A_{i}\right) P\left(B \mid A_{i}\right)}, \quad k=1,2,3, \cdots, n .
$$

The naive Bayes algorithm is based on Bayes rules. In many cases, the naive Bayes classification algorithm can be compared with ensemble learning and $K$-value nearest neighbor classification algorithms. Assuming that the attributes $X_{1}, X_{2}, \cdots, X_{n}$ are all conditionally independent of each other, this hypothesis $Y$ effectively simplifies the expression formula of $P(X \mid Y)$ and the estimated probability of the training data. In the case of $X=\left(X_{1}, X_{2}\right)$, there is a probability formula:

$$
P(X \mid Y)=P\left(X_{1} \mid Y\right) P\left(X_{2} \mid Y\right)
$$

When $X$ contains $n$ conditionally independent attributes, under the given hypothesis $Y$, there is a probability formula:

$$
P\left(X_{1}, X_{2}, \cdots, X_{n} \mid Y\right)=\prod_{i=1}^{n} P\left(X_{i} \mid Y\right)
$$

Suppose that $Y$ is an arbitrary discrete-valued variable, and the attribute $X_{1}, X_{2}, \cdots, X_{n}$ is an arbitrary discretevalued or real-valued attribute. For each new instance $X$ that it is required to classify, assign possible values of $Y$ and train a classifier that will produce a probability. $P$ represents the probability of $Y$ taking $k$ possible value, and the Bayesian rule is

$$
\begin{aligned}
P(Y & \left.=y_{k} \mid X_{1}, X_{2}, \cdots, X_{n}\right) \\
& =\frac{P\left(Y=y_{k}\right) P\left(X_{1}, X_{2}, \cdots, X_{n} \mid Y=y_{k}\right)}{\sum_{j} P\left(Y=y_{j}\right) P\left(X_{1}, X_{2}, \cdots, X_{n} \mid Y=y_{j}\right)},
\end{aligned}
$$

where $y_{j}$ is the sum of all possible values of $Y$; assuming that the characteristics of a given category $Y$ and $X_{i}$ are independent of each other, then

$$
P\left(Y=Y_{k} \mid X_{1}, \cdots, X_{n}\right)=\frac{P\left(Y=y_{k}\right) \prod_{i} P\left(X_{1} \mid Y=y_{k}\right)}{\sum_{j} P\left(Y=y_{j}\right) \prod_{i} P\left(X_{1} \mid Y=y_{j}\right)} .
$$


When $Y$ is the most probable value, it conforms to the naive Bayes classification rule:

$$
Y \longleftarrow \arg \max _{y_{k}} \frac{P\left(Y=y_{k}\right) \prod_{i} P\left(X_{1} \mid Y=y_{k}\right)}{\sum_{j} P\left(Y=y_{j}\right) \prod_{i} P\left(X_{1} \mid Y=y_{j}\right)} .
$$

Since the denominator in the formula does not depend on $y_{k}$, formula (6) can be simplified to

$$
Y \longleftarrow \arg \max _{y_{k}} P\left(Y=y_{k}\right) \prod_{i} P\left(X_{1} \mid Y=y_{k}\right)
$$

2.3. Sport Training. Sports training is an important part of competitive sports. It can be regarded as a course, a branch of science, and an academic classification, and its tendency is generally regarded as a comprehensive operational and applied discipline [15]. Sports training can improve the level of sports, and the active implementation of sports training can find and provide high-quality talent for national competitive sports, which has important practical significance in sports power [16]. After many years of improvement, the essence and system of sports training are constantly changing and progressing. Sports training has changed from the development of natural to the stage of development based on emerging technologies and multiamount of sports training and started the scientific training stage [17].

Adhering to the principle of physical and mental health in sports training can improve the health protection of coaches and athletes and avoid physical injury in the process of sport. This can not only help athletes maintain a healthy physique and follow regular sports training arrangements but also help athletes improve their wrong movements in sports training, improve their sport level, and create good competition results [18]. In sports training, the sports load borne by athletes will affect the effect of sports training. Proper exercise load can make the fitness of the body stronger. Once the exercise load is too low, it will not allow the body to achieve a certain level of energy and cause a stress reaction so that the training is invalid; when the load exceeds a certain level, the function of the body will be adversely affected, endangering the health of athletes [19]. The key to the successful innovation of sports training technology and tactics is to understand the development law of sports training technology and tactics. The innovation of organizational form can make the coaches' knowledge diversified, give full play to the collective wisdom, formulate a more scientific sports training plans, and improve the performance of sports training. The structural elements of sports training include training basis and preparation, training operation, and training support. The structure of sports training is the special relation between the elements of the training system, strengthening framework of the training system, and an open system.

2.4. Action Recognition. The banalization of the image is conducive to the further processing of the image, which makes the image simpler, reduces the amount of data, and can highlight the contour of the region of interest. The research of sensor network node system is the basis of large-scale sensor network research. The design and implementation of the node system greatly affect the function, performance, and investment cost of the entire network. Whether it is the traditional feature extraction method or the feature extraction method based on wavelet decomposition, it is necessary to manually design features for specific problems. Traditional image feature extraction is generally divided into three steps: preprocessing, feature extraction, and feature processing; and then, use machine learning methods to classify features and other operations. These features are not universal when the scene changes or the target performs different actions [20]. The main task of action recognition is to analyze the collected picture or video, identify the action sequence in the picture or video, use the computer to process the action in the video image, and finally achieve the purpose of positioning, tracking, and identifying the target in the video image through the computer. Video image processing system is a system that processes video images based on image processing algorithms. Action recognition is an important and challenging task, which is caused by the different styles and durations of executed actions [12]. Many action representation methods have been proposed to improve the performance of action recognition [21]. Since action sequences can be regarded as $3 \mathrm{D}$ objects in spacetime, we solve the problem of action recognition by recognizing $3 \mathrm{D}$ objects and characterize $3 \mathrm{D}$ objects by the probability distribution of local spatiotemporal features [22, 23]. Judging from the current research status, motion recognition technology can only collect human contours or movements based on the background environment that people set in advance or under the background of certain unfavorable factors, obtain the information and characteristics that we are interested in, and finally use a certain method or model to perform the final action recognition of the human body. In computer vision, action recognition refers to the action of classifying the actions present in a given video, and action detection involves locating the action of interest in space and/or time .

There are many methods of action recognition, including the following: (1) the method based on template matching, whose basic idea is to match the template to be classified with the template of known categories and realize the classification of the unknown categories through the similarity measurement between templates. (2) Based on the generation model method, this method uses the common probability function to determine the relationship between the observed attribute values and the action category information. (3) The motion recognition method used in this study is based on the method of generating model. Different movements need to train different models, and the parameters of each model can be obtained by learning the training data. In the process of classification, the features to be classified are input into the model of each category trained in advance, the matching degree with each model is calculated, respectively, and the matching category is selected as the category attribute of the action to be performed. 


\section{Construction of Recognition Model of Wrong Movement in Sports Training}

3.1. Data Acquisition. In this experiment, 12 members of a sports team were selected as the test objects; firstly, computer vision technology is used to collect the movement training action of the test object, and then, the hidden Markov model is used to recognize the video data; finally, the Bayesian algorithm is used to calculate and analyze the identified data and evaluate the experimental data. The basic information of the 12 test objects is shown in Table 1 .

According to the data in Table 1, we can see that the difference between the maximum height and minimum height of the team's 12 athletes is $5 \mathrm{~cm}$, the difference between the maximum weight and the minimum weight is $3 \mathrm{~kg}$, the difference between the maximum age and the minimum age is 4 years, and the difference of body mass index and training years is not big. Therefore, the influence of individual factors on sports training can be ignored.

3.2. Construction of Action Recognition Model. The action recognition model used in this experiment is a hidden Markov model, which enables us to guess the most likely next state through the apparent state, and is widely used in speech recognition, artificial intelligence, and action recognition. Hidden Markov model is a statistical model, which is used to describe a Markov process with hidden unknown parameters. The difficulty is to determine the hidden parameters of the process from the observable parameters. Hidden Markov model is composed of five parameters, which can be represented by five tuples or three tuples. There are five elements to determine five tuples: initial probability matrix, state transition matrix, observation probability matrix, number of states, and the number of observations corresponding to each state. In the representation of triples, the last two points are removed and the first three points are used to represent our model. Suppose $Q$ is the set of hidden states and $S$ is the set of possible observations corresponding to the hidden states, $n$ and $m$ in formula (8) denote all possible cases:

$$
\begin{gathered}
Q=\left\{q_{1}, q_{2}, \cdots, q_{n}\right\}, S=\left\{s_{1}, s_{2}, \cdots, s_{m}\right\}, \\
X=\left(x_{1}, x_{2}, \cdots, x_{T}\right), Y=\left(y_{1}, y_{2}, \cdots, y_{T}\right) .
\end{gathered}
$$

The state transition probability matrix of the model is as follows:

$$
\begin{gathered}
A=\left[a_{i j}\right]_{N \times N^{\prime}}, \\
a_{i j}=P\left(x_{t+1}=q_{j} \mid x_{t}=q_{i}\right) .
\end{gathered}
$$

The observation probability matrix of the model is as follows:

$$
B=\left[b_{j}(k)\right]_{N \times N},
$$

TABLE 1: Basic information table of test objects.

\begin{tabular}{lccccc}
\hline Number & $\begin{array}{c}\text { Height } \\
(\mathrm{cm})\end{array}$ & $\begin{array}{c}\text { Body weight } \\
(\mathrm{kg})\end{array}$ & Age & $\begin{array}{c}\text { Body mass } \\
\text { index }\end{array}$ & $\begin{array}{c}\text { Training } \\
\text { years }\end{array}$ \\
\hline 1 & 180 & 73.3 & 18 & 22.4 & 6 \\
2 & 177 & 73 & 22 & 23 & 7 \\
3 & 182 & 75 & 19 & 22 & 7 \\
4 & 182 & 72 & 20 & 21.5 & 9 \\
5 & 179 & 73 & 22 & 21 & 9 \\
6 & 181 & 75 & 21 & 20 & 7 \\
7 & 180 & 74 & 18 & 20.5 & 6 \\
8 & 178 & 74.5 & 19 & 22 & 8 \\
9 & 176 & 72.8 & 18 & 21 & 7 \\
10 & 181 & 72.7 & 19 & 21.6 & 7 \\
11 & 177 & 73.4 & 20 & 23 & 8 \\
12 & 182 & 74.1 & 20 & 23.3 & 8 \\
\hline
\end{tabular}

where $b_{j}(k)$ is the probability of generating observation $s_{k}$ under the condition of state $q_{j}$ at time $t$ :

$$
b_{j}(k)=P\left(y_{t}=s_{k} \mid x_{t}=q_{j}\right) .
$$

$\pi=\pi_{i}$ is the probability vector of the initial state, where $\pi_{i}$ is the probability of being in state $q_{i}$ at $t=1$ :

$$
\pi_{i}=P\left(x_{1}=q_{i}\right)
$$

Therefore, the three elements of the hidden Markov model are as follows:

$$
\lambda=(A, B, \pi)
$$

3.3. Human Motion Analysis Method Based on Sports Energy Flow. Motion energy flow is a time-domain difference detection algorithm based on image spatial features. When using this method, we first extract the spatial features of the moving image. Let the human moving image be $M$, the two-dimensional Gaussian function of the standard deviation $\delta$ is $G(\delta)$, the symbol $*$ represents the convolution operation, and the image $M$ can be decomposed into $d$ scale Laplacian pyramid description operator:

$$
\left\{S_{L}(M) \mid 0 \leq L \leq d\right\}
$$

When $L=0$, for $S_{L}(M)$,

$$
S_{L}(M)=M-M * G(\delta) .
$$

When $L>0$, for $S_{L}(M)$,

$$
S_{L}(M)=M * G\left(\delta^{L}\right)-M * G\left(\delta^{L+1}\right)
$$


TABLE 2: Error action statistics table.

\begin{tabular}{lccc}
\hline & $\begin{array}{c}\text { Number of } \\
\text { people }\end{array}$ & $\begin{array}{c}\text { Total error } \\
\text { frequency }\end{array}$ & $\begin{array}{c}\text { Personal error } \\
\text { frequency }\end{array}$ \\
\hline KV & 8 & $58 \%$ & $45 \%$ \\
FDOL & 9 & $50 \%$ & $30 \%$ \\
KLA & 11 & $33 \%$ & $28 \%$ \\
JF & 6 & $25 \%$ & $15 \%$ \\
TF & 5 & $17 \%$ & $10 \%$ \\
JA & 6 & $15 \%$ & $6 \%$ \\
\hline
\end{tabular}

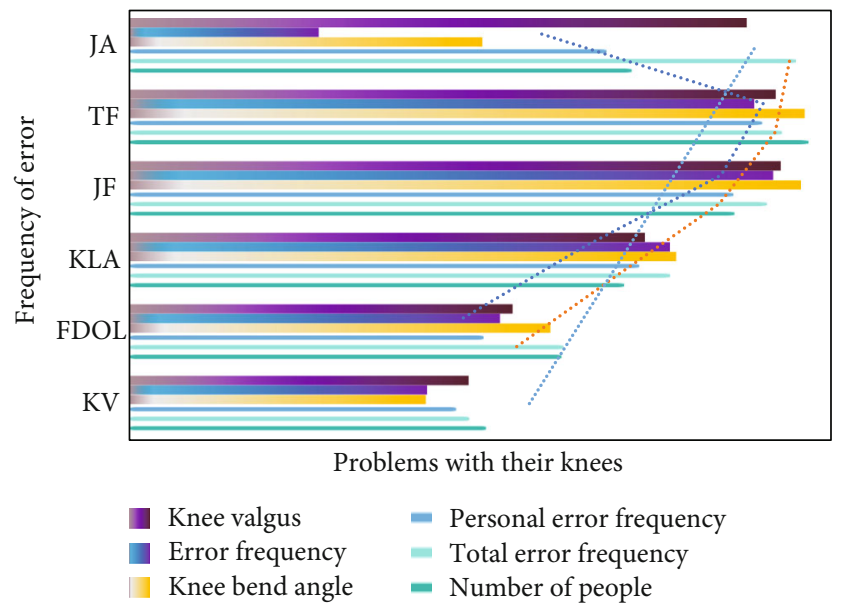

FIGURE 1: Knee flexion angle problem and the frequency of errors.

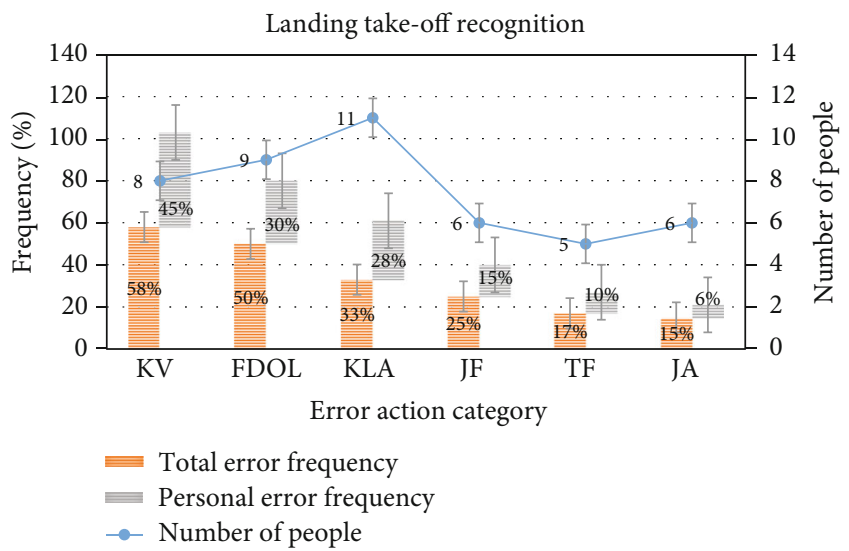

FIgURE 2: Error action recognition statistics chart.

The Laplacian pyramid feature of any pixel $(x, y)$ in the image can be expressed as

$$
\begin{gathered}
S_{L}(M)=G_{L}-G_{L+1}^{S}, \\
G_{L+1}^{S}(x, y)=\sum_{i, j} Y(i, j) G_{L}(x+i, y+j),
\end{gathered}
$$

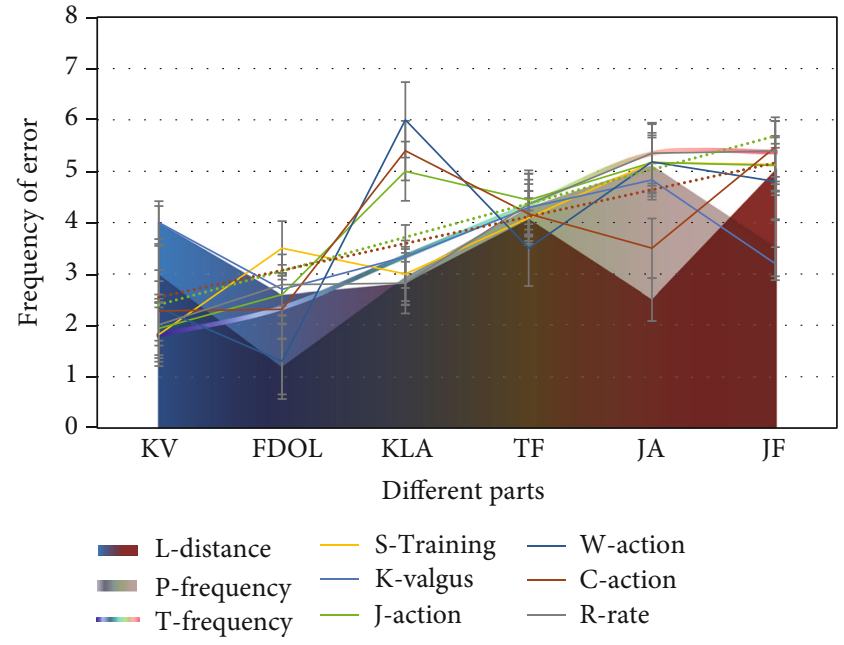

Figure 3: The correlation between footing frequency and error rate.

where $S$ represents the height, $L$ represents the length, $i$ represents the increase in the $x$ component, and $j$ represents the increase in the $y$ component.

$$
G_{s+1}(x, y)=G_{S}^{L}(2 x, 2 y) .
$$

$Y(i, j)$ in the formula is a Gaussian function. On the $L$ scale, the following energy conversion function is further established for the Laplacian pyramid feature $S_{L}(M)$ through a logarithmic function:

$$
T_{L}(M)=\ln \left|S_{L}(M)\right| G\left(\delta^{L+1}\right) .
$$

After energy conversion, since the value of $\left|S_{L}(M)\right|$ in many pixels is $0, s\left|S_{L}(M)\right|$ in formula (21) has

$$
\left|S_{L}(M)\right|=\left\{\begin{array}{l}
\left|S_{L}(M)\right|, S_{L}(M) \neq 0, \\
1, S_{L}(M)=0 .
\end{array}\right.
$$

Then, use the exponential function to construct the energy conversion map:

$$
\begin{gathered}
F_{L}(M)=\left|S_{L}(M)\right| Q_{L}(M), \\
Q_{L}(M)=\left\{\begin{array}{l}
e^{\lambda T_{L}(M)},\left|e^{\lambda T_{L}(M)}-e^{\lambda \rho}\right|>\varepsilon, \\
0, \text { other. }
\end{array}\right.
\end{gathered}
$$

$\lambda$ is the adjustment operator, and $\varepsilon, \rho$ is the infinitesimal and image quality parameters, respectively.

\section{Simulation Analysis of Wrong Action Recognition in Sports Training}

4.1. Athlete Wrong Action Recognition. The 12 athletes of the team were recognized for landing and jumping movements, and the types of wrong movements of the athletes as well as 


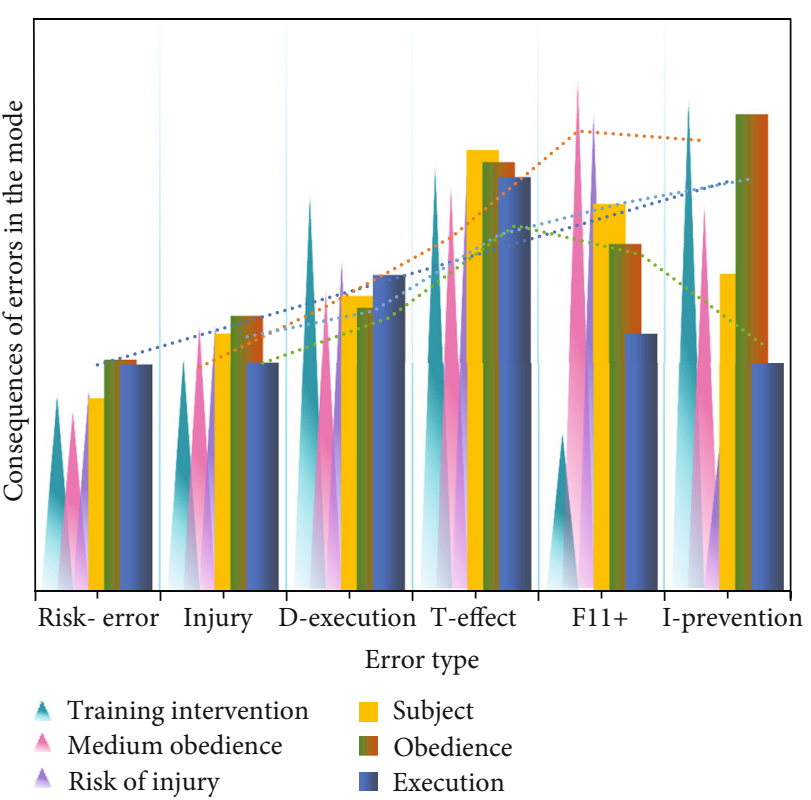

Figure 4: Effect of athlete execution degree on training effect in preventive training.

Body parts

L-metry L-sides M-index L-right L-length F-height F-length

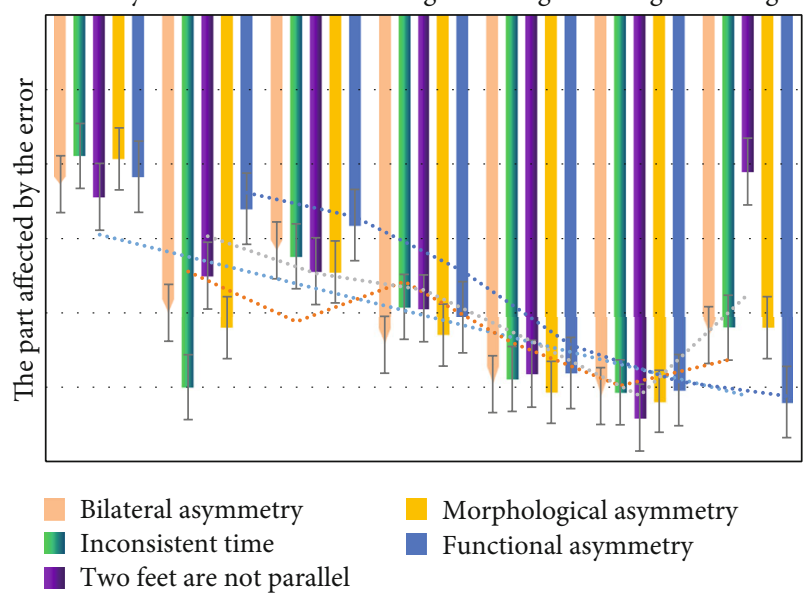

Figure 5: Inconsistent landing time on both sides or the two feet are not parallel.

the frequency of various wrong movements and the number of mistakes were counted. The types of known wrong actions include knee valgus (KV), distance between feet when landing (FDOL), knee flexion problem (KLA), joint flexion problem (JF), trunk flexion problem (TF), and joint angle problem (JA). Table 2 shows the final statistical results of the wrong actions of the 12 athletes.

As shown in Figure 1, the team has the largest number of knee bending problems, but the frequency of errors is relatively small. Knee valgus occurred most frequently in all wrong actions, and the frequency of personal errors reached $45 \%$.

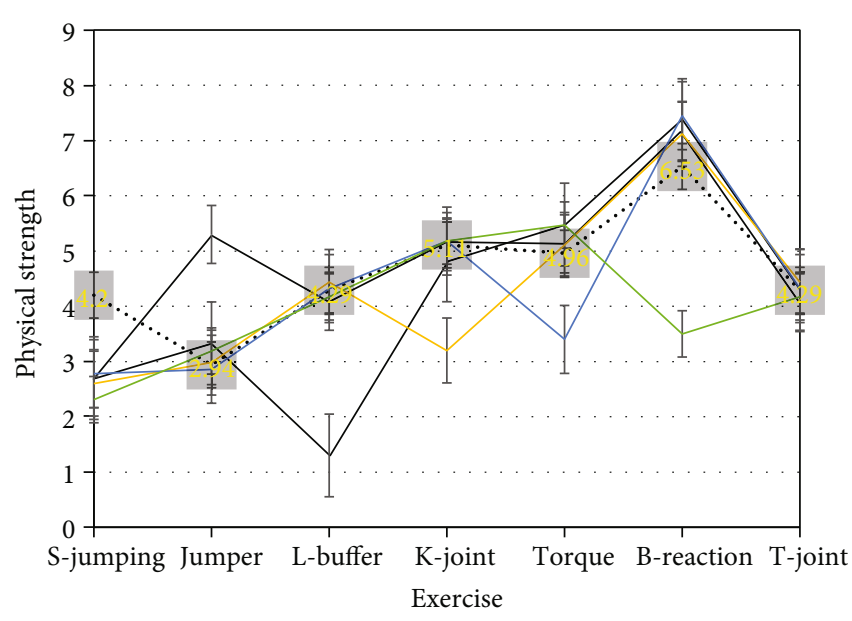

FIGURE 6: Landing buffer for emergency stop jumpers or step jumpers.

According to the data in Figure 2, we can see that the number of athletes who made mistakes in the knee bend angle problem in the landing jump of the team reached 11 people, the largest number of all wrong action categories. The frequency of the team's wrong actions was concentrated in the knee valgus, the total frequency of errors reached $58 \%$, and the frequency of personal errors reached $45 \%$.

As shown in Figure 3, the team landing distance problem also appeared more frequently, with a total frequency of $50 \%$ and a personal frequency of $30 \%$. It can be seen that athletes should pay more attention to the problems of knee valgus and the distance between feet when they are training.

As shown in Figure 4, the athletes' performance in injury prevention training plays a very critical role in the training effect. F11 injury prevention training intervention was conducted for young athletes. It was found that athletes with high obedience ability had a 35\% lower injury risk than those with medium obedience ability. The subjects' compliance and execution were very good.

As shown in Figure 5, the asymmetry of landing on both sides is mainly due to the inconsistent landing time on both sides or the two feet are not parallel when landing. The reason for this imagination may be related to morphological asymmetry or functional asymmetry. Morphological asymmetry of the lower limbs is a common phenomenon. There may be differences in morphological indicators of lower limbs on both sides of athletes. For example, there are certain differences in leg length, foot height, and foot length on the left and right sides.

\subsection{Characteristics of the Athlete's Body Center of Gravity} during Sports Training. As shown in Figure 6, in the landing buffer of an emergency stop jumper or a stride jumper, the knee joint needs to generate a certain moment to buffer the ground reaction force. The moment generated by the knee joint is opposite to the moment of the ground reaction force, thus ensuring balance during landing. However, in this process, the quadriceps muscle generates a moment and causes a forward shearing force on the end of the tibia. 
TABLE 3: Characteristic table of body center of gravity after successful action.

\begin{tabular}{lccc}
\hline & Displacement & Velocity & Acceleration \\
\hline Movement start & 0.36 & 0.28 & 1.19 \\
Ball off hand & 0.95 & 0.31 & 1.43 \\
Vertical shot & 1.64 & 1.58 & 0.91 \\
Swing & 1.82 & 0.84 & 1.66 \\
\hline
\end{tabular}

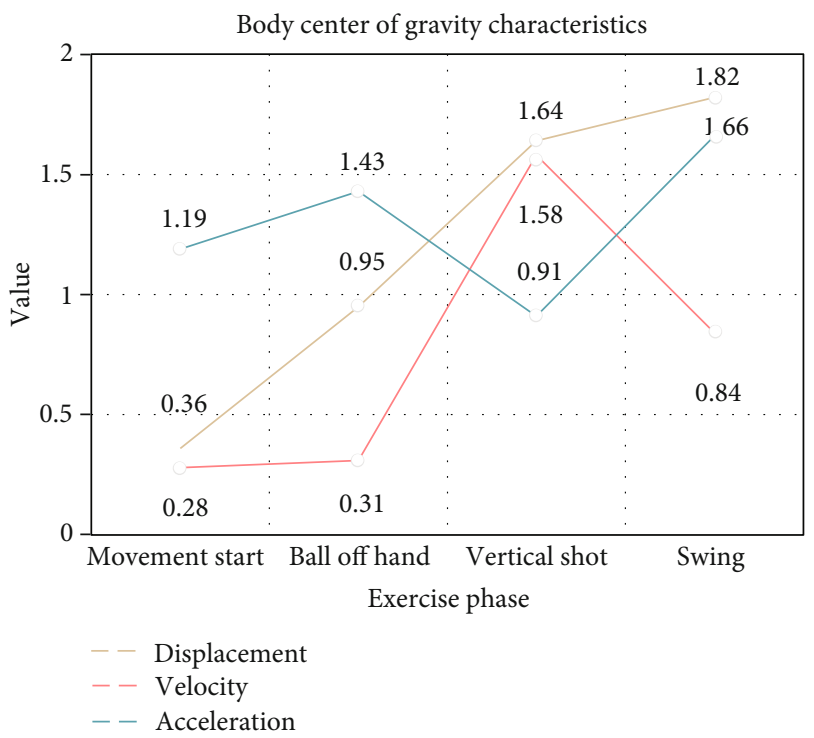

FIGURE 7: A statistical chart of the results of the characteristics of the body's center of gravity under the successful action.

TABLE 4: Body center of gravity characteristics after failed action.

\begin{tabular}{lccc}
\hline & Displacement & Velocity & Acceleration \\
\hline Movement start & 0.65 & 0.53 & 1.06 \\
Ball off hand & 1.21 & 0.48 & 0.49 \\
Vertical shot & 1.38 & 0.25 & 1.51 \\
Swing & 1.64 & 0.47 & 0.45 \\
\hline
\end{tabular}

Excessive movement of the knee joint in the sagittal plane will increase the shearing force of the knee joint.

Calculate the characteristic data of the displacement, speed, and acceleration of the body's center of gravity of the athletes of the team during the exercise training action of the badminton serve stage. The final statistical results are shown in Table 3.

According to the data in Table 3, we can see the characteristic data of the center of gravity of the athlete after successfully completing the movement. It can be seen from the table that from the beginning of the movement to the swing stage, the displacement of the athlete's body center of gravity has been increasing, and the speed of the vertical hit is the largest and the acceleration is the smallest. To better analyze the data in the table, we convert the data into a graph form, and the final result is shown in Figure 7.

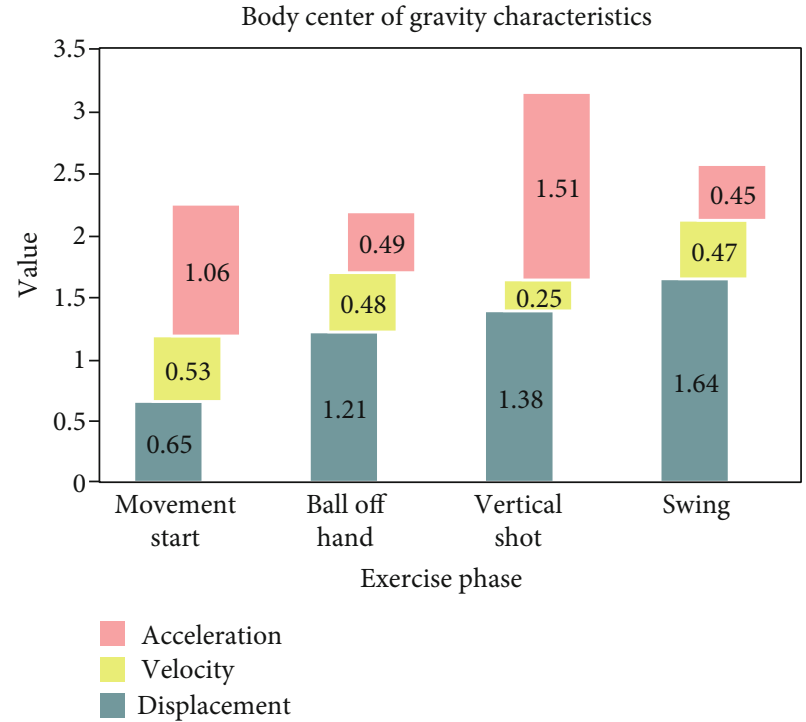

FIgURE 8: A statistical chart of the results of the characteristics of the body's center of gravity under the athlete's action error.

According to the data in Figure 7, we can see that when the athlete's sports training action is successful, the maximum displacement value of the athlete's body, center of gravity during swing is $1.82 \mathrm{~m}$, and the maximum speed of the body's center of gravity during vertical shooting is $1.58 \mathrm{~m} / \mathrm{s}$; the maximum acceleration of the body's center of gravity during swing is $1.66 \mathrm{~m} / \mathrm{s}^{2}$. Then, statistics characteristic data of the displacement, speed, and acceleration of the body's center of gravity when the athletes make mistakes during the exercise of the badminton serve stage. The final statistical results are shown in Table 4.

According to the sentences in Table 4, we can see the characteristic data of the body's center of gravity after the athlete has made a mistake. From the data in the table, we can see that the displacement of the body's center of gravity has been increasing, but the athlete's body's center of gravity velocity is the smallest and the acceleration is the largest when hitting vertically. We converted the table into a graph to facilitate the analysis of the data, and the final result is shown in Figure 3.

According to the data in Figure 8, we can see that, in the case of an athlete's movement training mistakes, the maximum displacement value of the athlete's body center of gravity when vertical shooting is $1.51 \mathrm{~m}$, the maximum speed of the body's center of gravity at the beginning of the exercise is $0.53 \mathrm{~m} / \mathrm{s}$, and the acceleration of the body's center of gravity is $1.64 \mathrm{~m} / \mathrm{s}^{2}$ when swinging. Combining the data in Figures 7 and 8 , we can see that there is little difference in the displacement of the athletes in the case of a successful serve action and a fault in the serve action, but there is a larger difference at the beginning of the movement and when the ball leaves the hand.

4.3. Accuracy Comparison of Action Recognition Models. To show that the action recognition method used in this research has certain advantages in action recognition, this 
Table 5: Accuracy comparison table.

\begin{tabular}{lccc}
\hline & $\begin{array}{c}\text { Method of this } \\
\text { article }\end{array}$ & $\begin{array}{c}\text { Deep learning } \\
\text { methods }\end{array}$ & $\begin{array}{c}\text { EMG signal } \\
\text { method }\end{array}$ \\
\hline 5 & $90 \%$ & $85 \%$ & $80 \%$ \\
10 & $93 \%$ & $84 \%$ & $83 \%$ \\
15 & $95 \%$ & $87 \%$ & $85 \%$ \\
20 & $97 \%$ & $89 \%$ & $89 \%$ \\
25 & $98 \%$ & $94 \%$ & $93 \%$ \\
30 & $98 \%$ & $97 \%$ & $95 \%$ \\
\hline
\end{tabular}

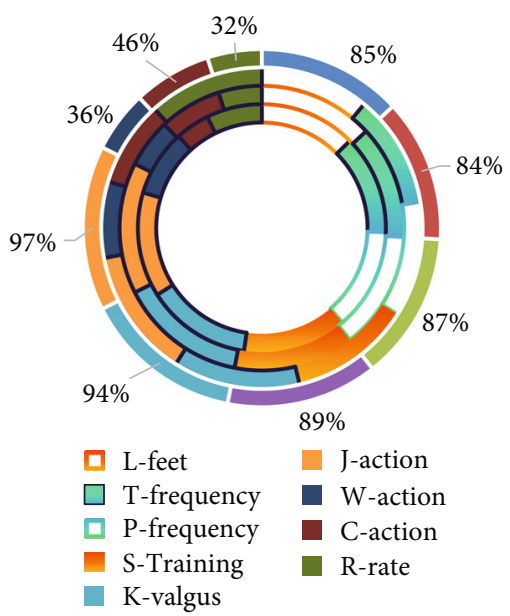

FIgURE 9: Action recognition accuracy after 30 experiments.

research compares and analyzes the action recognition method used in this paper with deep learning-based action recognition and muscle signal-based action recognition and counts 30 experiments. The accuracy of the action recognition of the next three methods and the final results are shown in Table 5.

To observe the changes in the data more intuitively, we converted the data in the table into a graph for analysis, and the final result is shown in Figure 9. According to the data in the figure, we can see the accuracy of action recognition of different action recognition methods after 30 experiments. According to the data in the table, we can see that the accuracy of the action recognition method used in this article is more than $90 \%$ after several experiments, and the more the number of experiments, the higher the accuracy. Among them, the electromyography signal (EMG) is the superposition of the action potential of the motor unit in many muscle fibers in time and space. Surface electromyography (SEMG) is a comprehensive effect of superficial muscle EMG and nerve trunk electrical activity on the surface of the skin, which can reflect neuromuscular activity to a certain extent.

According to the data in Figure 10, we can see that the accuracy of the motion recognition method used in this article has reached more than $90 \%$ in motion recognition, and the accuracy of the other two methods is less than $90 \%$ before the experiment performed 20 motion recognitions.

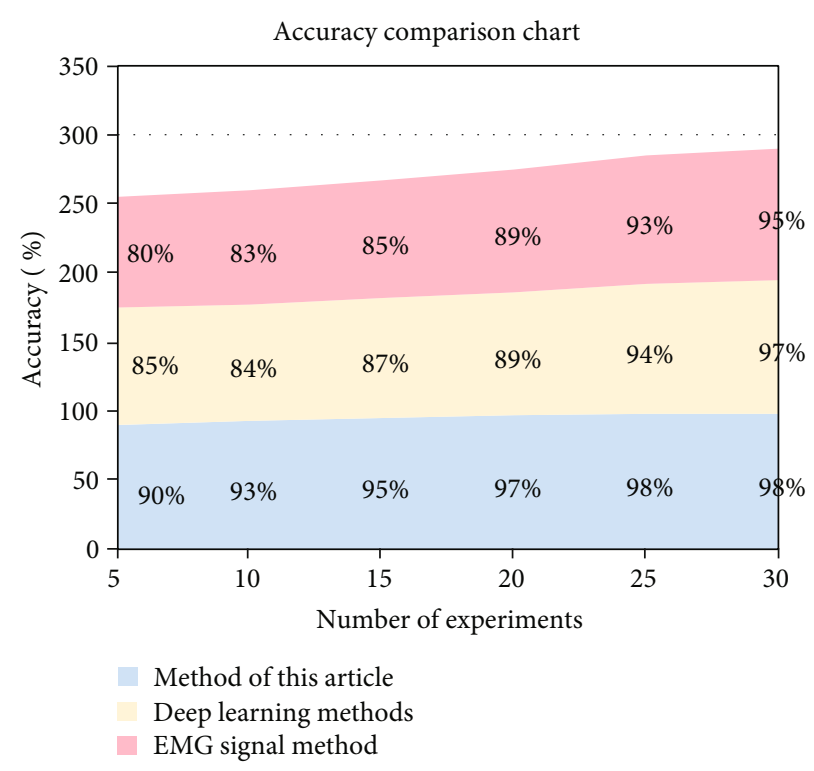

Figure 10: Comparison of accuracy of different action recognition methods.

And the accuracy is lower than that of the method used in this article. Therefore, the action recognition method used in this article has certain advantages in action recognition.

\section{Conclusions}

From the current development point of view, although motion recognition has been developed to a certain extent, it is still far from the expected goal. Nowadays, most of the researches in the field of motion recognition are still in the stage of simple action and simple background. Increasingly advanced recognition models and algorithms must be proposed and tried if we want to realize the accurate recognition of complex actions in a complex environment and finally realize the artificial intelligence of machines.

In this study, a hidden Markov model based on computer vision is constructed to identify the wrong movements of athletes in sports training, the computer vision technology is used to realize the video collection of the athletes' landing take-off action and badminton serve action, the hidden Markov model is used to identify the video data, the error frequency of take-off and the characteristics of body center of gravity in each stage of badminton serve action under the conditions of success and failure were counted, the Bayesian classification algorithm is used to calculate and analyze the identified data, and the accuracy of three different action recognition methods is compared and analyzed. The results of this experiment show that athletes should pay more attention to the problems of knee valgus and the landing distance of two feet in the landing and take-off movements. Computer vision embedded in experimental wireless communication can effectively identify the wrong actions of badminton players. It realizes the automatic learning of distinguishing features from the original data. Compared with manual design features, it saves time and manpower, and the accuracy of estimating the position and 
behavior of the human body and gestures is significantly improved. The embedded network can transform the original signal into the time-frequency domain for analysis and can provide information in the time and frequency domain of the signal at the same time and can describe the original measurement data from multiple aspects, so it contains more information. The transport layer considers that increasing devices will be connected to the network, and the limitations of traditional wired connection methods are becoming increasingly obvious. Connecting devices to the network is the key to the realization of data transmission and intelligence.

Although the experiment was carried out smoothly, there are still some deficiencies to be improved in some aspects. First of all, the experimental data of this study is not comprehensive enough, and the data analysis of various movements of athletes needs to be improved. Secondly, the accuracy of recognition is only considered in the comparative analysis experiment between this method and other action recognition methods; in the later experiment, we need to improve the experiment in this aspect and compare and analyze different methods from different levels. Finally, the hidden Markov model and Bayesian classification algorithm can be further improved.

\section{Data Availability}

Data sharing not applicable to this article as no datasets were generated or analyzed during the current study.

\section{Conflicts of Interest}

The author declares that there are no conflicts of interest.

\section{References}

[1] T. Nguyen Anh, T. Huynh-The, and K. U. Khan, "ML-HDP: a hierarchical Bayesian nonparametric model for recognizing human actions in video. Circuits and systems for video technology," IEEE Transactions on Circuits and Systems for Video Technology, vol. 29, no. 3, pp. 800-814, 2018.

[2] C. Zhang, Y. Tian, X. Guo, and J. Liu, "DAAL: deep activationbased attribute learning for action recognition in depth videos," Computer Vision and Image Understanding, vol. 167, pp. 37-49, 2018.

[3] Z. Liu, J.-t. Huang, and X. Feng, "Action recognition model construction based on multi-scale deep convolution neural network. Guangxue jingmi gongcheng/optics and precision," Engineering, vol. 25, no. 3, pp. 799-805, 2017.

[4] X. Liu, Y. Li, and Q. Wang, "Multi-view hierarchical bidirectional recurrent neural network for depth video sequence based action recognition," International Journal of Pattern Recognition and Artificial Intelligence, vol. 32, no. 10, article 1850033, 2018.

[5] S. Zhou, L. Chen, and V. Sugumaran, "Hidden two-stream collaborative learning network for action recognition," Computers, Materials \& Continua, vol. 63, no. 3, pp. 1545-1561, 2020.

[6] N. Jaouedi, N. Boujnah, and M. S. Bouhlel, "Deep learning approach for human action recognition using gated recurrent unit neural networks and motion analysis," Journal of Computer Science, vol. 15, no. 7, pp. 1040-1049, 2019.

[7] J. Li, X. Mao, X. Wu, and X. Liang, "Human action recognition based on tensor shape descriptor," IET Computer Vision, vol. 10, no. 8, pp. 905-911, 2016.

[8] S. Yu, Y. Cheng, S. Su, G. Cai, and S. Li, "Stratified pooling based deep convolutional neural networks for human action recognition," Multimedia Tools and Applications, vol. 76, no. 11, pp. 13367-13382, 2017.

[9] R. Cui, A. Zhu, J. Wu, and G. Hua, "Skeleton-based attention-aware spatial temporal model for action detection and recognition," IET Computer Vision, vol. 14, no. 5, pp. 177-184, 2020.

[10] Z. Yao, J.-Q. Lv, C. Xiang, Q.-Y. Cen, and X.-G. Tian, “Application research of computer vision in external quality inspection of agricultural products," Science and Technology of Food Industry, vol. 40, no. 14, pp. 89-93, 2019.

[11] C. Kanellakis and G. Nikolakopoulos, "Survey on computer vision for UAVs: current developments and trends," Journal of Intelligent \& Robotic Systems, vol. 87, no. 1, pp. 141-168, 2017.

[12] Z. Wan, Y. Dong, Z. Yu, H. Lv, and Z. Lv, "Semi-supervised support vector machine for digital twins based brain image fusion," Frontiers in Neuroscience, vol. 15, article 705323, 2021.

[13] V. Kolmogorov, Y. Boykov, and C. Rother, "Applications of parametric maxflow in computer vision," in 2007 IEEE 11th International Conference on Computer Vision, pp. 1-8, Rio de Janeiro, Brazil, 2007.

[14] B. Raman, S. Kumar, P. P. Roy, and D. Sen, "Proceedings of international conference on computer vision and image processing: CVIP 2016, volume 1," Advances in Intelligent Systems and Computing, vol. 1, no. 1, pp. 459-460, 2017.

[15] I. Fister, S. Rauter, X. S. Yang, K. Ljubič, and I. Fister Jr., "Planning the sports training sessions with the bat algorithm," Neurocomputing, vol. 149, pp. 993-1002, 2015.

[16] V. A. Zaporozhanov, T. Borachinski, and Y. N. Nosko, "Assessment of children's potentials in dynamic of initial stage of sport training," Journal of Physical Education \& Sport, vol. 15, no. 3, pp. 525-530, 2015.

[17] J. C. Redondo, E. Fernández-Martínez, and J. M. I. Velasco, "The effect of relative age in throwing disciplines of participants in the national sports training plan in Spanish athletics," Cuadernos de Psicologia del Deporte, vol. 19, no. 3, pp. 156$167,2019$.

[18] A. Boshnjaku, I. Dimauro, E. Krasniqi et al., "Effect of sport training on forearm bone sites in handball and soccer female players," Journal of Sports Medicine \& Physical Fitness, vol. 56, no. 12, pp. 1503-1505, 2015.

[19] S. Liang, "Intelligent decision model of sports training knowledge based on dynamic deep learning," Quarterly Journal of Indian Pulp and Paper Technical Association, vol. 30, no. 6, pp. 174-181, 2018.

[20] Z. Wu, Y. Huang, L. Wang, X. Wang, and T. Tan, “A comprehensive study on cross-view gait based human identification with deep CNNs," IEEE Transactions on Pattern Analysis and Machine Intelligence, vol. 39, no. 2, pp. 209226, 2017.

[21] H. A. Abdul-Azim and E. E. Hemayed, "Human action recognition using trajectory-based representation," Egyptian Informatics Journal, vol. 16, no. 2, pp. 187-198, 2015. 
[22] M. Chen, L. Gong, T. Wang, and Q. Feng, "Action recognition using lie algebrized Gaussians over dense local spatio-temporal features," Multimedia Tools \& Applications, vol. 74, no. 6, pp. 2127-2142, 2015.

[23] A. A. Adnan Salih and C. Youssef, "Spatiotemporal representation of 3D skeleton joints-based action recognition using modified spherical harmonics," Pattern Recognition Letters, vol. 83, pp. 32-41, 2016. 